\title{
¿Por qué volver a la escuela? Un estudio de caso sobre una institución de Educación de Adultos en Cuenca, Ecuador
}

Enviado: 28 de septiembre de 2020 / Aceptado: 15 de octubre de 2020

Publicado: 30 de diciembre de 2020

DANIEL SALINAS VILLACRÉS

Universidad Internacional de la Rioja

y Unidad Educativa Particular Universitaria de Azogues, Ecuador

daniel.salinas@ucacue.edu.ec

MARÍA ISABEL NEGRI CORTÉS

Departamento de Métodos de Investigación y Diagnóstico en Educación, Universidad Internacional de La Rioja, España mariaisabel.negri@unir.net

\section{RESUMEN}

La educación de adultos supone una segunda oportunidad de crecimiento formativo, laboral y social para aquellas personas que, por sus desventajas personales, económicas y socioculturales, se vieron abocadas a la deserción escolar. El objetivo de este trabajo fue conocer la experiencia de un grupo de alumnos de los programas de Educación de Adultos de una entidad educativa en Ecuador, indagando en los significados que adquiere para ellos acceder de nuevo al sistema educativo y en sus expectativas de futuro hacia el ámbito sociolaboral. Para ello, se recurrió a un enfoque cualitativo de investigación con el fin de construir conocimientos desde lo particular y subjetivo, estableciendo reflexiones y aproximaciones sobre las realidades que convergen en los programas de Educación de adultos. Asimismo, en el diseño de investigación se

\section{ABSTRACT}

Why going back to school? A case study on an Adult Education institution in Cuenca, Ecuador

Adult education is a second opportunity for formative, labor and social growth for those people who, due to their personal, economic and socio-cultural disadvantages, were forced to drop out of school. The objective of this work was to know the experience of a group of students of the Adult Education programs of an educational entity in Ecuador, investigating the meanings that accessing the educational system acquires for them and their future expectations in the socio-labor sphere. To do this, a qualitative research approach was used in order to build knowledge based on the particular and subjective, establishing reflections and approaches on the realities that 
optó por el estudio de casos que nos permite otorgar una perspectiva holística y contextual ante el sentido del fenómeno, utilizando para ello la técnica de la entrevista semiestructurada y el análisis documental. Contamos con una muestra de seis participantes que forman parte de un centro de enseñanza secundaria dirigida a esta población. A pesar de la escasez de investigaciones en la misma línea de este artículo, varios de los resultados obtenidos presentan similitud con las experiencias, significados y expectativas de personas que han aportado sus testimonios en otros estudios. Un resultado nuevo que aporta esta investigación a la literatura, se encuentra en la importancia que adquiere para los estudiantes convertirse en referentes para la trayectoria académica de sus hijos. Estas personas aún precisan enfrentarse a problemáticas socioeconómicas, por lo que se valen de los estudios para alcanzar mejoras materiales, así como a su inclusión social y la de sus familias.

Palabras Clave: educación de adultos, inclusión social, inclusión educativa, estudio de caso, investigación cualitativa. converge in Adult Education programs. Likewise, in the research design, the study of cases was chosen, which allows us to give a holistic and contextual perspective to the meaning of the phenomenon, using the semi-structured interview technique and documentary analysis. We had a sample of six participants who are part of a secondary education center aimed at this population. Despite the paucity of research along the same lines as this article, several of the results obtained show similarity with the experiences, meanings and expectations of people who have contributed their testimonies in other studies. A new result that this research contributes to the literature is found in the importance that it acquires for students to become references for the academic trajectory of their children. These people still need to face socio-economic problems, so they use their studies to achieve material improvements, as well as their social inclusion and that of their families.

Keywords: adult education, social inclusion, educational inclusion, case study, qualitative research.

\section{INTRODUCCIÓN}

Pensamos a la educación como un mecanismo clave en la búsqueda del desarrollo de individuos, familias, comunidades y sociedades. Gracias a ella, nos hacemos con destrezas, conocimientos, valores y aptitudes cognitivas, pragmáticas y sociales, que nos disponen a darle un sentido al mundo del que somos parte, y nos da la perspectiva necesaria para tomar un lugar en la realidad personal, laboral, ciudadana, cultural y social en que nuestra cotidianidad transcurre. Además de esto, a decir de Blázquez (2001), en la sociedad de la información marchamos hacia una época caracterizada por el imperativo de una constante actualización de los conocimientos que poseemos para mantenernos dentro de la sociedad, como también lo ratifican Romero y Hernández (2019).

En contraste, al estar la escuela atravesada en todo momento por la esfera de lo social, en su interior se siente el impacto de problemáticas más amplias, como la escasez de recursos 
económicos, el trabajo juvenil, la falta de capital cultural, la exclusión social (normalizada en una sociedad que fomenta la competitividad), la discriminación racial, la ruralidad, la desestructuración familiar, el menoscabo del valor social del estudio, por citar algunos de varios ejemplos, que se han convertido en las causas para que, estas desigualdades conduzcan al abandono de la escuela, dejando a niños y jóvenes al margen de aquel anhelado desarrollo (Romero \& Hernández, 2019).

En el caso particular del Ecuador, el estudio de Sánchez (2015) revela cómo la dinámica de la deserción escolar ha presentado una evolución favorable. En un lapso de veinte años, entre 1994 y 2014, la tasa de abandono escolar entre los menores de 18 años disminuyó del 6,6\% al 3,4\%. Históricamente, las dos mayores causas para que esto suceda en el país han sido, a nivel de los años de la primaria y primera mitad de la secundaria, la distancia física entre los hogares de los niños y las instalaciones de sus escuelas, seguida de motivos personales y familiares. Entre quienes cursaban el Bachillerato, predominan considerablemente causas de índole personal.

Panoramas como este, devienen en nuevas situaciones críticas para las personas afectadas y sus familias siendo ya adultos, tales como saber en riesgo la permanencia en sus empleos, verse obligados a tomar trabajos bajo circunstancias precarias, o en definitiva al desempleo de larga duración, dado el hecho de carecer de una cualificación mínima; factores que en suma, impiden a los sujetos salir de la situación originaria de pobreza que les condujo a la deserción educativa (García, Casal, Merino, \& Sánchez, 2013). Investigaciones como la de Martínez (2015), lo ponen en evidencia, revelando cómo para el año 2012 en España, partiendo del nivel de estudios alcanzado, las personas más afectadas por el desempleo fueron las analfabetas o quienes sólo habían terminado la primaria.

De la mano de las consecuencias laborales, no haber concluído los estudios básicos genera ciertas huellas psíquicas en en el desarrollo vital: sentimientos de fracaso, que a su vez degradan la autoestima y el concepto que tienen de sí mismos (Espinoza, Loyola, Castillo, \& González, 2014). Además está la desvinculación social, que junto a un capital cultural poco desarrollado, expone a las personas a ciertas situaciones de vulnerabilidad como la migración, la desestructuración de los valores que permiten la vida en sociedad, la incursión en las drogas o hasta la delincuencia (Ruiz-Ramírez, García-Cué, \& Pérez-Olvera, 2014).

Frente a realidades como esas, no sería de extrañar que muchas personas que habiendo abandonado la escuela en su niñez o adolescencia, bien por falta de interés hacia lo académico, y desconocimiento de las consecuencias sociales y laborales futuras de la escolaridad inconclusa; bien porque la situación que vivían no les daba otra opción, decidan darse una segunda 
oportunidad para culminar sus estudios. Para que esto sea posible, sería un error poner la responsabilidad de esa iniciativa únicamente sobre los hombros del individuo, parafraseando a Ozturk (2001), los gobiernos están en la necesidad y responsabilidad de elevar los años de estudio de su población a través de sus sistemas educativos, que actúan como garantes de la igualdad de oportunidades de formación entre todos sus ciudadanos (en especial entre aquellos que están expuestos a una mayor vulnerabilidad social): sin un buen nivel educativo, el desarrollo social y económico no es posible. Los avances industriales y tecnológicos, la innovación y el emprendimiento, son posibles entre personas que están bien capacitadas.

Y, ¿cuál es el nivel de años de estudio entre la medianía ecuatoriana? Dentro del país ha existido un incremento progresivo del promedio de escolaridad. Partiendo del año 2001, este se encontraba en 6,61 años. En el 2010 la cifra subió a 9,04 y cerrando el 2016 el país alcanzó un promedio de años de estudio de 10,13. Sobre el analfabetismo, la fuente oficial más reciente es de 2010, año en el que se hizo un censo poblacional que reveló que el 6,8\% de los ecuatorianos no saben leer ni escribir. De ese grupo, la etnia más afectada fue la indígena, que representó una presencia del 20,4\% (Instituto Nacional de Estadísticas y Censos, 2010; 2016).

\subsection{Sobre la deserción escolar}

Este fenómeno, al que también se denomina en la literatura como abandono escolar, o interrupción de la escolaridad, es comprendido como un alejamiento procesual y finalmente total de la escuela, que conlleva a cambios de la dinámica vital personal, ya que se modifican hábitos, emociones y cogniciones que el niño o joven tiene sobre sí mismo, así como en su dinámica familiar (Comisión Intersectorial de Reinserción Educativa (2006) citada en Espinoza, Loyola, Castillo, \& González (2014).

Algunos de los autores que han abordado esta problemática, consideran que está asociada a dos grupos generales de factores, que bien podríamos caracterizar como internos a la escuela, y otros externos a la misma.

En el primer grupo, el de los factores internos, encontramos prácticas ejecutadas por parte de los funcionarios escolares que favorecen la segregación de los alumnos. Entre ellas estarían un estilo autoritario de convivencia, respuestas educativas a la diversidad que terminan en exclusión, la enseñanza centrada en el docente que reduce a un segundo plano las inquietudes, espontaneidad y las necesidades relacionales y cognitivas del alumno. En una cotidianidad educativa de esta naturaleza, el capital cultural que lleva el menor a la escuela es anulado y la educación se convierte en una suma de pautas conductuales y sociales que hay que obedecer 
pasivamente (Herrera, 1999; Espíndola \& León, 2002; Ou, Mersky, Reynolds, \& Kohler, 2007; Rumberger \& Lim, 2008, Morentin Encina, Ballesteros Velázquez, \& Mateus, 2019).

Desde el aporte de los autores citados, además de este modo de relacionarse de los docentes, también hay vivencias en los propios estudiantes que impiden alcanzar una convivencia satisfactorio en la escuela y que se han hallado vinculados al su abandono: comportamientos conflictivos y vínculos negativos con docentes y otros pares, escasa participación de las actividades del colegio, absentismo escolar, bajo rendimiento académico y una baja adaptación a los diferentes subniveles educativos.

Referente al grupo de los factores externos a la escuela, priman las situaciones de carácter económico y social. Sánchez (2015) propone una importante relación entre los años de menor nivel del Producto Interno Bruto (PIB) ecuatoriano y las tasas de abandono escolar en el país, mismas que mostraron índices más altos. En los años de mejora del PIB nacional y de mayor inversión estatal en educación, la deserción escolar se redujo.

A escala familiar, una economía limitada y la necesidad de iniciarse laboralmente en edades tempranas, son señales de alarma para la decisión de dejar la escuela, con lo que, la generación de los hijos, que carecerá de niveles formativos adecuados y por lo tanto tendrá menos oportunidades de acceder a empleos dignos, heredará las desigualdades sociales que atravesaron sus padres (Espíndola \& León, 2002; Espinoza, Loyola, Castillo, \& González, 2014; Ruiz-Ramírez, García-Cué, \& Pérez-Olvera, 2014; Sánchez, 2015).

Condiciones sociales, como la violencia y la disfuncionalidad del núcléo familiar, la presencia de adicciones entre los miembros del hogar, el embarazo adolescente, o la monoparentalidad, representan factores de vulnerabilidad que podrían predisponer a una situación de abandono escolar. Mención enfática merece también la disponibilidad de un acervo cultural escaso, en el que se hace una valoración negativa entre los progenitores del menor frente a los estudios, o porque ellos mismos tienen un logro de años de estudio por debajo de los mínimos. Entre otras potenciales causales se encuentran problemas de salud persistentes, discapacidades, o la misma baja motivación del alumno (Espinoza, Loyola, Castillo, \& González, 2014; Espíndola \& León, 2002; Sánchez, 2015).

Romero y Hernández (2019), agrupan estos factores dentro de las categorías de endógeno y exógeno desde el punto de vista del propio estudiante. La tipología endógena abarca los aspectos más intrínsecos al sujeto (actitud, autoconcepto, salud, discapacidad, motivación, rendimiento, entre otras), así como su círculo familiar y social de mayor influencia (estructura y clima familiar, estatus y cultura familiar, grupos de pares, conflictos, etc.). Lo exógeno es 
aquello que supera el control del propio menor y no está en sus manos: la escuela, su clima y su praxis, la política educativa, la valoración social de la educación, la sociedad, la situación laboral y económica regional. Todos estos factores operan en una estrecha relación que requiere de soluciones globales.

\subsection{Sobre el regreso a la escuela}

A raíz del abandono escolar, la persona se encuentra en medio de dos posibilidades: la primera es que en definitiva se mantenga en esta ausencia y no vuelva a ser parte del sistema educativo, a esta condición, se le llama escolaridad inconclusa. Pero no todos aquellos que se distanciaron de la escuela lo hacen para siempre, y en ese sentido, la segunda posibilidad reside en que, después de unos años, los estudios vuelvan a ser parte del proyecto de vida de la persona, y decida retornar al colegio en una edad que no necesariamente es la que legislativa y socialmente se adscribe para determinado subnivel educativo. A estos casos se les conoce como rezago escolar (Sánchez, 2015).

Para quienes se encuentran en esa segunda situación, existen ofertas educativas que cubren los niveles de alfabetización, educación básica, básica superior (en modalidad intensiva) y bachillerato (en modalidad intensiva) diseñadas específicamente para que jóvenes y adultos en edad post-escolar retornen y progresen hacia la culminación de su formación (Ministerio de Educación, 2020a). Cabe especificar a modo referencial, que en su modalidad ordinaria, la Educación General Básica en Ecuador comprende diez grados (que van de los cinco a los catorce años de edad) a los que siguen tres años de bachillerato (Ministerio de Educación, 2020b).

La educación de adultos, al igual que cualquier iniciativa encaminada hacia la formación, está fundamentada sobre una serie de actividades de aprendizaje estructuradas, organizadas y sujetas a una planificación. Estas acciones varían en su grado de formalidad, con diversas ofertas curriculares, metodologías de enseñanza-aprendizaje o grados académicos que van desde los años básicos, hasta niveles profesionales y superiores. Ante cualquiera de estas particularidades, la educación de adultos apunta en su objetivo al fortalecimiento integral de conocimientos, potencialidades y aptitudes específicas entre sus acreedores, de modo tal que se enriquezca su potencial para ser parte activa del desarrollo material y social de su entorno. (Espinoza, Loyola, Castillo, \& González, 2014; Sarrate, 1997).

Acerca de las ofertas formativas en los grados de educación primaria, secundaria y bachillerato dirigidos a adultos, Merriam \& Brockett (2007) los definen como programas enfocados en el desarrollo de conocimientos y capacidades elementales, interrumpidas en cierto periodo del desarrollo infantil o de la adolescencia, debido a una salida precoz del sistema escolar. Históricamente, estos programas han sido además concebidos desde la perspectiva de lo com- 
pensatorio, como una formación de segunda oportunidad con función remedial, si bien no se la ha dejado de pensar como integral (Sarrate, 1997).

Según Martínez (2015) la formación de secundaria de adultos ha de ser reflexionada desde su lugar periférico a los márgenes de los escenarios educativos, relegada a un segundo plano. Esto, por una parte, debido a que a nivel de sociedad, dotamos de mayor prestigio a quienes culminan los niveles primario, secundario y de bachillerato en el marco de las edades normativas; y por otra parte, esta ubicación poco protagónica se explica por el hecho de que el presupuesto, las infraestructuras y el equipo profesional y técnico de los centros que forman adultos, representan una parte bastante minoritaria de los sistemas de educación. Otro rasgo definitorio de la educación de adultos según el mismo autor consiste en que en las salas de clase de estos planteles, convive una amplia diversidad de edades.

Cabe finalizar este basamento conceptual considerando que los programas de formación de adultos tienen en cuenta las particulares necesidades, motivaciones, idiosincrasia y concepciones de sí mismo y del mundo que posee el adulto y que lo diferencian del niño o del adolescente, factor que apenas en las décadas más recientes ha sido incorporado, puesto que tradicionalmente, para la formación de personas adultas se practicaba apenas una emulación de lo que los docentes practicaban en los colegios ordinarios con sus alumnos más jóvenes (Fernández, 2000). La educación de adultos además, responde a una estructura y cultura institucional y que en un contexto más amplio, responde a un contexto interpersonal, socioeconómico, político e histórico (Foley, 2020).

Una institución que se ha establecido como referente de la educación para la población adulta al sur del Ecuador, es la Unidad Educativa "Promoción Social Integral del Austro"1 asentada en la localidad de Cuenca (Ecuador). En este contexto se desarrolló la investigación que compone de estas páginas, la cual forma parte del Trabajo Fin de Máster de Salinas (2019), que más ampliamente, trazó diversos recorridos sobre el abandono temprano de la escuela y el retorno de las personas adultas a la misma, planteándose reflexiones psicopedagógicas sobre las implicaciones personales, sociales, familiares y laborales que esto conlleva.

\subsection{Planteamiento del problema}

A pesar de la relevancia de la educación de adultos para los sistemas educativos de cada país, este fenómeno ha recibido poca atención en lo que concierne al ámbito investigativo, quedan- 
do relegado a un segundo plano en contraste con lo que sucede con la educación ordinaria. La inclusión del tema en la academia, podría traducirse en la posibilidad de mejorar las ofertas educativas que beneficien directamente a los estudiantes adultos, de cara a su desempeño personal, afectivo, sociofamiliar, y laboral. Con la intención de aportar a su estudio, los autores nos proponemos dar respuesta a la pregunta: ¿por qué deciden las personas adultas reincorporarse al sistema educativo y cómo viven su regreso?

De esta problemática, se desprenden los objetivos trazados para el estudio, que fueron: a) Describir las vivencias de los estudiantes en su retorno al colegio, b) Conocer los significados que atribuyen a su educación, y c) Determinar sus expectativas laborales y académicas para el futuro.

\section{MATERIAL Y MÉTODO}

\subsection{Diseño de la investigación}

La investigación partió desde un enfoque cualitativo, en la búsqueda de hacer posible el encuentro con las subjetividades de los participantes, a través de sus propios discursos. Se empleó como diseño de investigación el estudio instrumental de caso, con la expectativa de aprehender los fenómenos particulares que seleccionamos en toda su complejidad y singularidad hasta alcanzar su comprensión, puesto que un caso representa un sistema delimitado, con elementos que tienen sus propios límites, pero que revelan su mismidad (Stake, 2010).

Adicionalmente, con el estudio de caso buscamos la comprensión integral, mediante la descripción del sentido del fenómeno. Con ello, se ha apuntado a destacar los sucesos clave de las vivencias de los participantes en su faceta educativa, gracias a lo cual, pudimos acceder a la elaboración de una teoría emergente que reflexione sobre su unicidad y complejidad particular. Dentro de esta metodología se recogen diferentes fuentes y técnicas de recogida de datos y se concibe un aspecto contextual, que es el horizonte desde el cual reflexiona a los atributos estudiados (Álvarez \& San Fabián, 2012).

\subsection{Contexto y Selección de los informantes}

El contexto seleccionado para la elaboración de nuestro estudio de caso fue la Unidad Educativa Particular Promoción Social Integral del Austro, ubicada en la ciudad de Cuenca, que ofrece formación en los subprogramas correspondientes a la educación secundaria en el Ecuador, 
esto es Educación General Básica en subnivel Superior, y Bachillerato General Unificado, dirigido específicamente a jóvenes y adultos en condición de rezago escolar. Su labor educativa contempla el fomento de la autoformación, al combinar tutorías presenciales y virtuales en una oferta de estudios semipresencial, adaptándose a la disponibilidad de tiempo de sus alumnos, condicionada por las jornadas laborales que la mayoría de ellos tiene.

Una cantidad considerable de su población proviene de la ruralidad y en muchos casos sus condiciones laborales no gozan de estabilidad, y se encuentran en situación de subempleo, influidos por la carencia de una formación que permita su promoción.

La selección de los informantes consistió en la aplicación de un muestreo probabilístico estratificado. A través de esta técnica se buscó garantizar la obtención de aportes procedentes de sujetos que, si bien son parte de la misma población, presentan diversidad entre sus características, enriqueciendo de ese modo, la información recogida para el análisis a nivel de heterogeneidad de significados y experiencias. La muestra fue seleccionada en base a tres estratos: grupos etarios (de entre 18 y 24 años, 25 a 40 años y de 40 años en adelante). Otro estrato fue el género, para contar con una muestra equilibrada entre hombres y mujeres, y finalmente el subnivel educativo: trátese de básica superior y de bachillerato.

Al momento de llevar a cabo la recogida de información, el plantel estaba conformado por 702 alumnos que se distribuían entre diferentes cursos y subniveles. De este total, se conformó una selección de seis estudiantes. Con el fin de salvaguardar su identidad, se otorgó a cada persona una codificación (A1, A2, A3, A4, A5 y A6).

\subsection{Procedimiento y Análisis}

La estrategia de recogida de información seleccionada para efectuar esta investigación fue la entrevista en profundidad, a la que se dio un diseño ad hoc y se compuso con seis preguntas guía. Al respecto, se ha tomado la sugerencia de Stake (2010) según la cual, en el diseño de entrevistas es preferible confeccionar un número corto de preguntas para el tema en el que se ha de investigar.

Adicionalmente se realizó un análisis documental sobre archivos referenciales sobre la historia y la comprensión que la institución hace de sí misma, análisis que permitió comprender la información obtenida de las entrevistas en relación a su contexto de referencia. Tras la recogida de los testimonios de cada participante, fue posible efectuar una interrelación o triangulación de la información generada, en aras de obtener un nivel propicio de fiabilidad para la información. 
Volviendo a las entrevistas, éstas fueron registradas en una grabadora de audio tras consentimiento de los participantes, y su duración fluctuó entre los 22 y los 34 minutos. Las preguntas aplicadas fueron semiestructuradas, y en ellas se abordaron cuatro temáticas: a) su realidad social y laboral, b) las motivaciones por las que retornaron al colegio, c) sus percepciones y significados acerca del programa de estudios y el hecho mismo de educarse, y d) sus proyectos a futuro en las facetas académica y laboral.

Las entrevistas a profundidad fueron aplicadas de forma individual y presencialmente en una sola sesión para cada caso, y se utilizó el espacio del departamento de orientación del plantel. Debido al hecho de que existía ya una familiaridad previa por parte de los estudiantes con el investigador, el proceso de socialización y generación de confianza para responder a las preguntas de la entrevista fue positivo.

Con el fin de garantizar la fiabilidad de estos datos, se les aplicó una triangulación, contrastando los testimonios que aportaron los diversos sujetos para darles un sentido en su contexto. Tras el registro de la información, fue necesario estructurarla con el fin de ubicar, recuperar y analizar los datos. Se partió por la transcripción de las entrevistas disponibles en audio a un procesador de textos, que representaban un conjunto de datos inicialmente heterogéneo, que gradualmente fue tratado y organizado tomando como base un sistema de precategorías, o lo que es lo mismo, criterios para agrupar la información definidos de antemano. Estas categorías fueron: experiencias en el retorno al colegio, significados de la educación, y proyecciones al futuro.

\section{RESULTADOS}

Tras recoger los discursos facilitados por las personas que participaron en las entrevistas, ponemos a disposición del lector los hallazgos obtenidos, y que fueron especialmente valiosos para dar respuesta a los planteamientos de este artículo.

\subsection{La vivencia del retorno al Sistema Educativo}

Tomar la decisión de volver a estudiar cuando los años de la adolescencia ya han pasado, es una fuente de angustia que dispara sentimientos de inseguridad y temor frente a la valoración que los otros pueden hacer de nosotros, y a la autoimagen que nos formamos. El componente social adquiere una trascendencia considerable para afianzar la idea de que estudiar ha sido una buena o una mala elección, puesto que al propiciarse el encuentro con otras personas con 
las que los estudiantes se pueden sentir identificados, cuestiona tales temores. Encontrar a personas en un momento histórico similar al de su desarrollo vital, fortalece el surgimiento de procesos afectivos favorables para el desempeño académico individual.

"Yo me imaginaba a mis compañeros así jovencitos; pero cuando llegué, vi a varios que ahora son mis
compañeros, de mi edad, de más edad, y fue también algo que me hizo sentir bien, decir no soy sólo yo,
hay más gente que está intentando también" (A5).

La base del sentimiento de identificación es la que edifica la vivencia de comunidad, que los estudiantes ponen de manifiesto al momento de hablar de su experiencia educativa. Se expresan sentimientos cálidos como la empatía y la confianza, y valores como la solidaridad y el cuidado. En los alumnos de educación de adultos entrevistados, el colegio les ha ofrecido la oportunidad de construir amistades valiosas, que refuerzan su permanencia en el mismo, amplía sus redes sociales y mejora su sentimiento de pertenencia y su autoestima.

\footnotetext{
"Me gustó, me sentí como si estuviera en mi familia, con amigos... hay mucha relación con todos los compañeros, conversamos con todos, tenemos una buena relación... son ya maduros, conversamos de la vida, de la familia" (A3).
}

"No puedo bajar (el rendimiento) por lo mismo que uno siente ese aprecio" (A5)

Pero aquellas son vivencias que no sólo se dan con los pares de la clase. Los entrevistados han dejado ver la cercanía que han establecido con el profesorado, relación que se basa en la búsqueda del docente por responder a su situación real. Su experiencia del retorno a los estudios ha sido positiva, y otro de los factores para que esto sea así, viene de este hecho. Describen a sus profesores como accesibles, disponibles, amistosos, comprensivos de sus contingencias particulares, entre las cuales ha de concebirse que, para ellos, ser estudiantes es apenas una faceta más, que se equilibra entre las de cónyuges, hijos, padres o madres de familia y de trabajadores. Estas tres facetas se influyen mutuamente en sus vidas y ellos manifiestan que un docente flexible y que sabe contener, es un agente valioso para su formación, sin basarse en relaciones de autoridad o de poder.

\footnotetext{
"La institución para mí es más como una familia... es como que uno es dueño aquí, uno se siente parte, familia, querido" (A1).

"Más que todo amigos, y claro, siempre respetando esa parte de licenciado" (A4).
} 
“Ellos nos comprenden muchas veces por las situaciones que pasamos, nos apoyan” (A5).

"A veces realmente el tiempo no da, no avanzamos a hacer algún trabajo, ustedes nos han sabido comprender o explicar, o mandarnos una tarea extra para que hagamos en la casa. Eso está muy bien por parte de los maestros, que nos entiendan... no nos han tratado mal, han sido comprensivos. Nos han ayudado entre los deberes, cuando no podemos algo nos han indicado, han sabido escucharnos los problemas que tengamos" (A6).

Resulta también llamativa la apreciación que hacen de los contenidos de sus aprendizajes, mismos que son valorados en función de su utilidad práctica, sea que puedan incidir positivamente sobre sus competencias laborales, parentales, sociales o personales. De esto, se vuelve fundamental pensar en la necesidad de ofrecer currículos flexibles para la educación de adultos (en el contexto ideal de una legislación educativa apropiada), que abarquen la posibilidad de traducirse en resultados directos sobre la experiencia cotidiana del colectivo de los estudiantes adultos.

\footnotetext{
“Tengo mi hijo que tiene 4 años, entonces necesito saber algo para su escuela, y me sirve de mucho lo que aprendo aquí para poder ayudarle a él” (A1).

"En el trabajo ahora es diferente, sé cómo hablar, antes no podía ni hablar, me atragantaba y ahora tengo reuniones todos los lunes y me dicen: usted ha cambiado bastante, ¿qué está aprendiendo?” (A3).

"Todos los contenidos, y lo que cada uno de los licenciados nos explica, es una base fundamental... tengo a mis hijos, cualquier pregunta, uno ya está empapado de conocimientos” (A4).

"Cada vez que yo hablo algo, alguna cosa me acuerdo acá de lo que estoy aprendiendo, digo: esto aprendí, esto hice hoy día” (A6).
}

Hemos podido percibir cómo los entrevistados valoran su retorno al sistema educativo, en términos de la gratificación social que esto les produce. La literatura citada en apartados anteriores ha dejado ver cómo en los años tempranos del desarrollo, la falta de valor otorgado al estudio al interior del hogar suele estar asociada al abandono escolar. Inversamente, el apoyo anímico que los alumnos reciben por parte de sus padres, parejas y/o hijos en el núcleo familiar en el presente, representa una fuente de inspiración de cara a culminar la secundaria.

\footnotetext{
"Mi mami ha sido un gran apoyo para mí, tenga o no tenga, ella siempre me ayuda, siempre ve el bien por mí” (A1).
} 
"Mi esposa es laboratorista clínica, ella también me ayudó, dice: estudia porque es bueno, te va a servir de mucho" (A3).

"Gracias al apoyo de mi esposa que también hay bastante ese empuje que es muy importante, me motiva para seguir adelante" (A4).

"Por mi edad yo dije: o sea me van a decir que no, no estudies porque ya estás vieja; pero obtuve todo lo contrario de mi familia, me apoyaron a pesar de mi edad... realmente mi mamá y mis hijos son el motor para yo estar acá” (A5).

Pero no todos los participantes cuentan con ese apoyo incondicional de su círculo familiar y social. En un caso, algunos miembros de la red social de una estudiante buscaron impedir que ella culmine el bachillerato, aduciendo que su único proyecto vital debería ser la maternidad. Ella sin embargo, ha demostrado resiliencia, lo que le ha hecho capaz de sobreponerse a la presión negativa que recibió y ha conseguido la autoafirmación.

"Por la separación que hubo entre mi esposo y yo, hubo muchos problemas, me querían bajonear para que yo no estudiara... si yo no me salía de estudiar, me iban a quitar a mi hijo... otro obstáculo que también tenía, era la familia de mi mami, le decían: no le mandes a estudiar... ella ya tiene un hijo, ¿y por qué va a estudiar?, debe dedicarse al hijo y nada más” (A1).

Los entrevistados también han contemplado en sus reflexiones, además de sus oportunidades y motivaciones, algunas circunstancias que problematizan en mayor o menor medida su permanencia en los programas académicos. El primero de esos obstáculos es la disponibilidad limitada de tiempo para estudiar. Dado que gran parte de sus horas en el día transcurren entre el trabajo y el ejercicio de la parentalidad, su tiempo destinado a actividades formativas está distribuido entre las noches y los fines de semana, lo cual habla de un alto nivel de autoexigencia y de motivación hacia el estudio.

"El tiempo es enemigo de cada persona, quisiera que el reloj se pare y no siga... paso hasta altas horas de la noche haciendo mis cosas" (A4).

"Sí es complicado porque hay que saber dividir el tiempo, hay que saber estar completamente con los hijos, queda un tiempo pequeño para el estudio, pero siempre hay que darse un espacio... a veces me llamaba el médico los domingos, entonces yo le decía que no puedo porque yo los domingos estudio, entonces me dejó de llamar a las cirugías, yo no podía por el colegio" (A6). 
Otra de las limitaciones con la que se encuentran, es la inestabilidad de su situación económica, que responde a su dificultad para acceder a empleos fijos, lo cual va muy de la mano con la mismidad de su situación como personas con rezago escolar. Esto dispara las alarmas de la posibilidad de un nuevo abandono, que deberá ser contrarrestado con mecanismos eficientes por parte de la política educativa y la gestión de los centros.

\footnotetext{
“Me afecta ver que mis papás están sufriendo, más que todo son temas económicos” (A2).

"A veces quería ya no venir por lo económico, pero se pudo" (A5).
}

\subsection{Los significados de educarse en la adultez}

Para los entrevistados, la educación no tiene un sentido único. De entre las varias significaciones que ellos han aportado, hemos hallado tres subcategorías que encierran percepciones con elementos comunes entre ellos. De este modo, algunos significados apuntan a la resolución de necesidades internas; otras al fortalecimiento de los roles parentales y sociales; y otras de orden pragmático, laboral y material. Esto nos permite notar cómo la educación es en suma el medio por el cual se amplía la gama de posibilidades y recursos que enriquecen la vida de los individuos y que además beneficia a aquellos con los que co-existen. Comentaremos entonces aquellos significados organizados en sus categorías.

Partiendo de los significados asociados a la resolución de necesidades internas, percibimos que para los participantes estudiar es una actividad con la que miden su capacidad para auto-trascenderse, ir más allá de la situación personal fáctica en que se encuentran. De esto podemos observar que la educación les da mayor confianza en sí mismos, la resiliencia necesaria para sentirse capaces de afrontar vivencias desfavorables. Transmiten de diversas formas que la educación les permite autoafirmarse, ganar el coraje de ser ellos mismos. El sentimiento de eficacia que ganan desde lo académico se amplía a las demás facetas de sus vidas.

\footnotetext{
"Me ha servido en parte para dejarme de tantas cosas, para no estar así en la casa, yo qué sé, haciendo otras cosas" (A2)

"Yo me siento seguro de mí mismo, siempre con las metas propuestas fijas, yo sé que en la vida hay que subir escalón por escalón para lograr el éxito y en eso estoy” (A4).

"Fue cuando me separé que dije: no me voy a quedar así, si yo me separo quiero superarme... soy un poco inconformista, por más que saque una nota alta digo: no es lo suficiente, me falta más... siempre
} 
estoy diciendo: no me conformo fácil, puedo dar más. Si me equivoqué en algo, trato de corregirlo para la próxima hacerlo mejor" (A5).

"No hay que estar simplemente pensando que no puedo, hay que decir sí puedo día a día. A mí me han pasado muchas cosas en la vida y no me he dado por vencida, siempre he estado: sí puedo, voy a salir, no me voy a quedar ahí, ya me pasará” (A6).

Otro significado que apunta también a lo intrínseco, se relaciona con la necesidad que sentimos los humanos de adquirir cultura, de comprender el mundo físico y social del cual somos parte, lo cual incide directamente con la forma en que nos relacionamos con él. Estudiar sería la forma de engrandecer ese acervo con el que tratamos de responder a las preguntas sobre la vida que nos formulamos.

\footnotetext{
“A mí me motivó más por el aprendizaje... no sabía qué era física, qué era química. Escuchar esas palabras me incentivaron para aprender más... se conocen cosas nuevas, que antes no sabíamos, cuál es el significado de una palabra” (A1).

"Por ejemplo lengua y literatura, le hacen ver las cosas de diferente manera, a veces uno se dice, yo converso y digo lo que digo; pero no es suficiente, siento que detrás de eso, hay una forma de decir, ver el porqué de nuestras expresiones" (A5).
}

Pasando a la siguiente subcategoría, en la que hemos identificado el ámbito de la alteridad, hallamos dos significados que entrelazan a la educación con el fortalecimiento de sus relaciones con los demás. El primero viene de reconocer a los estudios como una vía para apoyar a sus hijos en sus estudios. Les brinda la oportunidad de ser un modelo de estudiante comprometido que deja una herencia simbólica y cultural, así como de jugar un papel activo en la adquisición de los aprendizajes, ya que al reforzar los contenidos del currículo con sus niños y adolescentes en casa, también los afianzan para sí mismos. Los discursos muestran cómo surge una proximidad en la relación con los hijos, lo cual tiene gran valor para ellos, así lo deja ver el hecho de que todos los participantes que tienen hijos hayan expresado esta significación, lo demuestra.

\footnotetext{
"Tengo mi hijo que tiene 4 años, entonces necesito saber algo para su escuela, y me sirve de mucho lo que aprendo aquí para poder ayudarle a él” (A1)

"Mi hijo ya está estudiando en la escuela y necesita que yo le enseñe algunas cosas... me muestra un libro de lenguaje y yo nunca había visto ese texto, esa materia y digo: y ahora, ¿cómo era esto?” (A3).
} 
"Mi primer muchacho está en tercero de bachillerato... es una alegría que él me venga a preguntar problemas de física” (A4).

“Tengo mi hija, ella está en el mismo curso que yo, tiene 14 años... le digo: mira, me saqué tal nota; y me dice: ¡wow mami! A veces me siente un poquito mal porque le gano a ella en notas... es bastante responsabilidad para uno... hasta la pequeñita, ella está en primer grado, llego a hacer los deberes y coge todas sus cosas y me interrumpe, ella no sabe escribir, se pone a copiar cada letrita de lo que yo pongo sin saber qué pone” (A5).

"He aprendido también cosas que me han ayudado para que yo pueda ayudar a mis hijos, antes no podía ayudarles en muchas áreas" (A6).

El segundo significado en esta subcategoría va más allá de la intimidad del círculo familiar. Contar con la secundaria completa representa un ascenso en el estatus que la persona proyecta dentro de la esfera social: sobreponerse a la marginalidad para adherirse a las nuevas configuraciones de la sociedad de la información. Ser reconocido por las personas del entorno de un modo nuevo y valioso, significa también cambios positivos en la idea que tenemos de nosotros mismos, incide sobre nuestra identidad.

"Tenía la mentalidad de ser alguien en la vida, poder llegar a ser alguien importante, reconocido, ya sea por mi dibujo o por cualquier otra cosa” (A2).

"Que mi hijo y mi mujer vean que yo he prosperado y he seguido adelante con mi esfuerzo" (A3).

"Estar al tanto de la ciencia, que está evolucionando, para no ser una persona aislada ante la sociedad” (A4).

"Hay mucha familia y amistades más que me dicen: oye qué chévere que estés estudiando, qué lindo, a pesar de que tienes hijos, sal adelante, un día te queremos ver graduada. Hay mucha gente que le ven a uno como que ya no se va a quedar atrás, que también va a salir adelante” (A6).

Existió una tercera subcategoría de significados para la educación, que la concibe como un recurso de utilidad en la consecución de objetivos laborales y materiales. Es una fuente de habilidades que elevan las aptitudes y el desempeño en el trabajo, en lo técnico, lo comunicativo y lo relacional. Con esto, amplían sus expectativas laborales, y de ingresos, cuestión muy deseada por un colectivo que en muchos casos debe lidiar con el subempleo y la informalidad. 
“Ahora ya sabemos más o menos de qué manera tratar a las personas” (A1).

"Hay personas que le dicen hasta aquí, muchas gracias, ¿por qué? Por no tener los conocimientos necesarios. Es el empuje que a uno le hace decidir prepararse" (A4).

“Me ha servido mucho este estudio porque sé cómo expresarme más claro a los médicos que están alrededor de mí trabajando, he sabido usar la computadora para contabilizar medicinas. Todas las áreas que me dan en esta institución me están sirviendo para el trabajo” (A6).

Algunas de las personas entrevistadas, debido a su trayecto laboral, poseen amplios años de experiencia en relación a su ocupación. Esto les ganó hacerse con saberes que, por no tener una formación básica, no pueden certificar, lo que se convierte en una desventaja de cara a un mercado de trabajo en el cual las instituciones y empresas dan preferencia a quienes presentan estudios formales. La educación se convierte en un medio para validar esos conocimientos acumulados gracias a la experiencia.

"En el transcurso que estaba en otro país, adquirí una profesión, sé el trabajo y no tengo el respaldo, el papel... tengo ya mi taller de carpintería, lo que me falta solamente es el título, el papel” (A4).

"Lo que yo quiero es llegar a ser licenciada, con los conocimientos que tengo, sé que voy a llegar allá... voy a seguir estudiando para llegar a la meta que yo quiero, no se me hará difícil porque ya tengo conocimientos" (A6).

Por último, en el ámbito laboral, la creación de emprendimientos también es una posibilidad deseable, para la cual se posiciona a los estudios como una instancia previa, que les dota de una mejor preparación, en miras de actualizar proyectos a mediano y largo plazo. De ese modo la motivación para formarse es alta, porque representa el futuro material de la persona y de su familia.

"Siempre mi sueño, mi ideal era yo vestirme con mi uniforme, asistir a una oficina, tener mi propia oficina... entonces era siempre lo que yo decía, debo estudiar para poder tener mi propio negocio" (A5).

\subsection{Proyecciones al futuro}

En lo referente al futuro académico y laboral, los estudiantes contemplan diversas opciones. En cinco casos, se evidencia la intención de ingresar a la educación superior, pero en distintos 
grados de concreción. En un caso, alcanzar los estudios superiores es una meta formal, hacia la cual la estudiante tiene el plan de dirigirse.

"Me decidí a estudiar para llegar a ser profesional en el área, mi sueño es ser licenciada en hospitalización y enfermería” (A6).

En los demás casos, esta intención parecería más bien tratarse de un anhelo ideal, antes de que de un proyecto conciso. Sin embargo, plantean como una meta más formalizada, la consecución de emprendimientos, iniciar negocios propios que den tranquilidad financiera a su núcleo familiar.

"Me gusta la carrera de finanzas, es mi sueño, es mi anhelo" (A1).

"Estoy tratando de sacar una propia marca de ropa, de diseños. En cuanto a mis estudios, tengo un cuñado que es arquitecto y me ha motivado a seguir esa carrera” (A2).

"Tendré mi taller de carpintería, fabricaré muebles, y quién quita, mi esposa administrando el negocio... Como mi esposa también es bachiller, tenemos pensado que cuando sea también bachiller, ir los dos juntos a la universidad" (A4).

"Yo quiero tener mi negocio propio... mi sueño sí es seguir la universidad, aunque a veces lo veo muy lejano; pero quiero seguir, quiero tener mi propio trabajo, quiero ser una profesional” (A5).

En otro caso, el entrevistado se proyecta hacia una meta a corto plazo: el formar parte de itinerarios de perfeccionamiento ocupacional.

"Mi objetivo principal es terminar de estudiar y de ahí ingresar a algún curso de electricidad o electrónica" (A3).

Resulta oportuno apreciar que, aunque los planes a futuro revelados sean divergentes entre ellos, en todos se busca el progreso laboral, de modo que consigan una mejora material para ellos y para los miembros de su familia. 


\section{DISCUSIÓN}

En el camino de la reflexión sobre los ámbitos que dan forma a la educación de adultos, las voces de los estudiantes poseen capacidad de aportar perspectivas valiosas sobre la mismidad de estos programas, de modo que se pueda evaluar el verdadero nivel de respuesta desde la política y las instituciones en relación a las vivencias, necesidades y expectativas de los alumnos que los conforman. Al igual que Morentin Encina, Ballesteros Velázquez, y Mateus (2019), consideramos que incluir sus testimonios, es la vía deseable hacia la democratización de la educación.

Teníamos como primer objetivo describir las vivencias de los estudiantes en su retorno al colegio, sobre el mismo, los entrevistados la han relatado en gran medida desde la dimensión del co-estar. Los estudios les han permitido crear redes sociales conformadas por amistades significativas en las que se sienten sostenidos por el grupo, y al que ellos también ofrecen su disponibilidad y apoyo. Un hallazgo similar se obtuvo en el estudio de Romero y Hernández (2019), en el que se sentía a los pares como una familia. Los estudiantes que participaron del estudio de Espinoza et al. (2014), rescatan la aceptación y el sostenimiento afectivo que dan y que reciben de sus amistades de la clase.

En lo relativo a la relación estudiante-docente, los jóvenes investigados por Morentin Encina, Ballesteros Velázquez y Mateus (2019), narraban que en varios casos un factor que les determinó al abandono temprano del sistema educativo fue una actitud desatenta y distante en el profesorado. Ellos señalaban la necesidad de tener profesores sensibles a sus circunstancias, problemáticas, capacidades e intereses, más allá de estrictamente ceñirse a cumplir con el currículo. Estudiantes que años después decidieron retornar a los planteles educativos, como los del presente artículo, o los de otras investigaciones (Espinoza et. al, 2014; Feito, 2015), han visto como un factor de permanencia justamente el vincularse con docentes que no se posicionan frente a ellos desde la verticalidad, sino que sus relaciones se fundamentan sobre la confianza, la simpatía y la apertura que ellos les transmiten. El docente, además de impartir contenidos, asume el rol de motivador, y a esta relación se le adjudican cualidades como la distensión, la voluntariedad y la fluidez, hechos esenciales para afirmar su continuidad en la educación.

El papel que juega la familia en el recorrido académico es determinante para el estudiante, tanto para operar a su favor como en su contra, sea en la niñez, en la adolescencia o en la adultez. Personas con trayectorias de abandono escolar han referido la necesidad de la implicación de los padres en los tramos educativos tempranos, siendo llamados a brindar apoyo y seguimiento en torno a la evolución de sus representados (Morentin Encina, Ballesteros Velázquez 
y Mateus, 2019). Ya como adultos, el sostén anímico de la familia es asumido como una oportunidad para progresar en los estudios, así se puede apreciar en esta investigación y de modo similar en la de Espinoza et. al. (2014). No obstante, en esta última, parecía que la percepción de apoyo familiar cobra aún más valía entre los alumnos de edades avanzadas, mientras que en nuestra investigación la presencia de la familia es vista como fundamental, sin importar si se trata de adultos jóvenes o de quienes están en la adultez media.

Los obstáculos que estos alumnos encuentran en la actualidad, derivan de problemáticas económicas, personales y/o familiares que inciden sobre su motivación y han sido superados en base a su capacidad de resiliencia; sin embargo, consideramos fundamental que la política educativa dirija su atención sobre estos sucesos, con el fin de evitar nuevos fracasos. Otra problemática hallada por este y por otro estudio (Feito, 2015), se relaciona con la limitación en el tiempo que estas personas tienen para los estudios, lo cual muchas veces se traduce en jornadas sobrecargadas, distribuidas entre el trabajo, el hogar y el colegio.

Otro objetivo de este estudio, fue conocer los significados que atribuyen a su educación. Los participantes viven su formación como una oportunidad de crecimiento en diversas áreas que abarcan lo afectivo, cognitivo, cultural, familiar, social, laboral y material. Un aporte novedoso hecho por ellos, y que no parece estar presente en otros estudios, es significar a los estudios como una oportunidad de volverse guías de los estudios de sus hijos, sentir que son modelos para ellos, y que además los pueden orientar en sus aprendizajes. Esto simboliza la asunción de un compromiso que muchos de los progenitores de los hombres y mujeres de este colectivo no realizó para con ellos. Del paso de una generación a otra, el acervo del capital cultural familiar se amplía.

Respecto a sí mismos, educarse en la adultez significa trascenderse a uno mismo al superar las condiciones personales iniciales que la vida le ha dado a cada persona. El sentimiento de confianza que produce el logro del progresivo éxito académico, alcanza las demás áreas de su vida, lo cual les permite autoafirmarse, no ser una consecuencia mecánica de las condiciones extrínsecas. Han mejorado su autoestima; y cognitivamente, han logrado una comprensión del mundo a la que antes no habían podido acceder. Estos hallazgos no son exclusivos de nuestra investigación, pues también se replican entre los discursos recogidos en el estudio de Espinoza et al. (2014).

Las personas también esbozamos nuestra identidad al relacionarnos, en la mirada que recibimos de los demás. La carencia de una formación educativa obligatoria va de la mano con la exclusión, la existencia en la periferia; pero al adquirirla, los estudiantes la superan en 
términos de estatus social (saberse reconocidos por su familia y la comunidad) y de promoción laboral (mejor desempeño en el trabajo, acceso a mejores plazas). Perciben que el colegio les brinda habilidades sociales, comunicativas y técnicas, gracias a las cuales sintonizan con la colectividad, la relación con el contexto se renueva en el acceso a nuevas esferas sociales. Así lo viven también los sujetos de otras investigaciones (Espinoza et al., 2014).

El estudio realizado por Feito (2015) establece para el caso de España, un alza en la demanda del servicio de los planteles que forman adultos en coincidencia con un período de incremento en las tasas nacionales de desempleo, en el contexto de la crisis económica que afrontó el país en el año 2008. Este factor nos permitiría reforzar la concepción de que una motivación para volver a los estudios es la búsqueda de la estabilidad material. Bajo la misma significación, encontramos en nuestros participantes que gracias a la culminación de la formación básica, certifican los conocimientos que han adquirido de manera directa a través de su historia laboral.

Por otra parte, también se retorna al sistema educativo con proyecciones a mediano y a largo plazo en lo académico y lo laboral. Relativo a nuestro tercer objetivo (determinar expectativas laborales y académicas a futuro), los participantes de otros estudios (Espinoza et al., 2014) se trazan como meta futura, poder acceder a mejores trabajos. Para nuestros entrevistados, una posibilidad deseable es la del emprendimiento de negocios familiares o de enriquecer la formación ocupacional a corto plazo. Una coincidencia directa hallada con el estudio citado, es la ambivalencia ante los estudios universitarios: en algunos casos se la plantea como una meta clara, mientras que en otros se trata de algo deseable, que se queda en el plano de la idealidad.

\section{CONCLUSIONES}

Los autores creemos que, incluir las voces de aquellas personas que tras una considerable espera han retornado a los sistemas educativos de sus países, conforme a sus propias experiencias, resulta fundamental en búsqueda de fomentar su promoción educativa. Otorga un conocimiento real acerca del tipo de formación que requieren, sus motivaciones, obstáculos y factores de permanencia, para en base a aquello propiciar la reflexión sobre el ajuste de los currículos, de los métodos de enseñanza y de legislación educativa, que generalmente son decididos en base a una distribución del poder que raramente escucha a los estudiantes.

Los participantes por su parte, valoran la vivencia de volver al colegio desde la función socializadora que cumple en sus vidas. Es para ellos valioso sentirse identificados con otras 
personas que vienen de trayectorias de vida parecidas, pero especialmente de construir vínculos sociales con sus compañeros de clase, de mantener relaciones cercanas con un profesorado que les brinda contención y apertura, así como de darse cuenta, a través de los estudios, de que reciben el apoyo de su círculo familiar. A la luz de este conocimiento, valdría preguntarse en qué medida los docentes, al igual que las familias son conscientes de la trascendencia que su actuación tiene en la experiencia de los estudiantes.

Su proceso de estudios actual, se ve así mismo enfrentado a diversos obstáculos que ponen a prueba su motivación y su resiliencia, pero que también deberían ser gestionados por los centros y la normativa educativa, tales como buscar medidas que ayuden a reducir la sobrecarga del estudiantado, ofrecer apoyos psicosociales de cara a promover la mejora de estrategias de afrontamiento y de habilidades para vida, así como trabajar en miras a lograr que los déficits socioeconómicos no lleven una vez más a estos estudiantes a verse excluidos de los centros educativos.

De entre los distintos significados que nuestros entrevistados dan a la educación, hemos hallado uno que destaca por su frecuencia, y que no ha estado presente en otros estudios: el de poder volverse guías de la educación de sus hijos, saberse responsables de transmitirles un legado cultural permanente. En relación al futuro, su meta es alcanzar la estabilidad material, con la cual puedan garantizar las bases de un desarrollo favorable a sus familias. Se trata, al final, de superar la exclusión social a la que sus circunstancias les han expuesto, tomando a la educación, como pieza protagónica de su accionar.

\subsection{Limitaciones y Prospectiva}

Debido a la temporalización de la investigación desarrollada (sujeta a los plazos de elaboración del TFM de Salinas, 2019), la muestra utilizada en este estudio de investigación podría presentar algunas limitaciones en su generalización a otros contextos, por lo que sería preciso contar con un mayor número de informantes para contrastar sus vivencias y dotar de mayor objetividad y rigor científico a este estudio. Igualmente, convendría utilizar otras técnicas y fuentes de recogida de datos, además de la entrevista a estudiantes, tales como la entrevista de profesionales, registros de observación de la práctica educativa o encuestas a las familias de los estudiantes adultos, con el fin enriquecer el proceso de triangulación de la información, que se traduzca en la amplificación de la confianza en los resultados. Por otra parte, se podría abordar esta investigación desde el ámbito de la educación comparada, contrastando las realidades de la educación de adultos en los contextos ecuatoriano y español, con el fin de reflexionar sobre las posibles mejoras y aportes que se podrían intercambiar entre ambos contextos. 
Respecto a las futuras líneas de intervención, consideramos de especial interés trabajar desde los centros de educación de adultos en el desarrollo de programas de educación emocional, que permitan fortalecer la autoestima y el autoconcepto de los estudiantes, para un mayor empoderamiento y autodeterminación en la búsqueda de progreso personal, laboral y social.

Por otra parte, dada la connotación social que existe en la actualidad sobre este tipo de programas, consideramos oportuno promover la sensibilización y concienciación social hacia una revalorización de los adultos que se reincorporan hacia el sistema educativo. Es decir, trascender la perspectiva de "desertores", "malos estudiantes", "personas sin oportunidades", hacia una consideración de personas resilientes, responsables y comprometidas, con afán de crecer y desarrollarse plenamente en el ámbito personal y laboral. Finalmente, con el objetivo de mejorar las concepciones socioeducativas sobre los estudiantes adultos y dar a conocer sus potencialidades y destrezas en el ámbito laboral, sería necesario facilitar la creación de programas de empleabilidad con empresas e instituciones, que contribuirán a la creación de mayores oportunidades laborales.

\section{REFERENCIAS BIBLIOGRÁFICAS}

Álvarez, C., \& San Fabián, J. (2012). La elección del estudio de caso en investigación educativa. Gazeta de Antropología, 28(1), 1-12. Recuperado de http://digibug.ugr.es/handle/10481/20644

Blázquez, F. (2001). La sociedad de la información y de la comunicación. Reflexiones desde la educación. En J. d. Extremadura, Sociedad de la Información y Educación (págs. 13-28). Mérida: Javier Felipe S.L. Recuperado de http://www.ub.edu/prometheus21/articulos/obsciberprome/ blanquez.pdf

Espíndola, E., \& León, A. (2002). La deserción escolar en América Latina: un tema prioritario para la agenda regional. Revista Iberoamericana de educación(30), 39-62. Recuperado de https://rieoei. org/RIE/article/view/941

Espinoza, O., Loyola, J., Castillo, D., \& González, L. (2014). La educación de adultos en Chile: experiencias y expectativas de los estudiantes de la modalidad regular. Última Década (40), 159-181. Recuperado de https://scielo.conicyt.cl/scielo.php?script=sci arttext\&pid=S0718-22362014000100008

Feito, R. (2015). La experiencia escolar del alumnado de la ESO de adultos. Un viaje de ida y vuelta. Revista de la Asociación de Sociología de la Educación, 8(1), 44-56.

Fernández, O. (2000). Especificidad de la Educación de Adultos. Bases psicopedagógicas y señas de identidad. Educación XXI, 3(1). Recuperado de http://revistas.uned.es/index.php/educacionXX1/article/view/406 
Foley, G. (Ed.). (2020). Understanding adult education and training. Routledge.

García, M., Casal, J., Merino, R., \& Sánchez, A. (2013). Itinerarios de abandono escolar y transiciones tras la enseñanza secundaria obligatoria. Revista de educación(361). Recuperado de http://www.educacionyfp.gob.es/dctm/revista-de-educacion/ articulosre361/re36103.pdf?documentId=0901e72b8163106d

Herrera, M. (1999). Fracaso escolar, códigos y disciplina: una aproximación etnográfica. Última Década(10). Recuperado de http://www.redalyc.org/pdf/195/19501012.pdf

Instituto Nacional de Estadísticas y Censos. (2010). El censo informa: Educación. Quito: INEC. Recuperado de http://www.ecuadorencifras.gob.ec/wp-content/descargas/Presentaciones/capitulo educacion_censo_poblacion_vivienda.pdf

Instituto Nacional de Estadísticas y Censos. (2016). Encuesta nacional de empleo, subempleo y desempleo. Quito: INEC.

Martínez, J. (2015). La educación secundaria para adultos y la FP de grado medio: ¿una segunda oportunidad en tiempos de crisis? Revista de la asociación de de Sociología de la Educación, 8(1), 2843. Recuperado de http://fes-sociologia.com/files/congress/11/papers/2272.pdf

Merriam, S., \& Brockett, R. (2007). The profession and practice of adult education. San Francisco: JosseyBass.

Ministerio de Educación. (2020a). Ministerio de Educación. Recuperado el 23 de Septiembre de 2020, de Educación General Básica: https://educacion.gob.ec/educacion general basica/

Ministerio de Educación. (2020b). Ministerio de Educación. Recuperado el 23 de Septiembre de 2020, de Acerca de ofertas Todos ABC: https://educacion.gob.ec/ofertas-todos-abc/

Morentin Encina, J., Ballesteros Velázquez, B., \& Mateus, S. (2019). ¿Igualidad de oportunidades? Más que el mero acceso: narrativas de jóvenes con trayectorias de fracaso y abandono temprano de la educación en España y Portugal. Revista Fuentes, 21(2), 143 - 159.

Ou, S., Mersky, J., Reynolds, A., \& Kohler, K. (2007). Alterable predictors of Educational attainment, Income and Crime: Findings from an Inner-City Cohort. Social Service Review, 81(1). Recuperado de https://www.jstor.org/stable/10.1086/510783?seq=1\#page scan tab contents

Ozturk, I. (2001). The role of education in economic development: A theoretical perspective. fournal of Rural Development and Administration, 33(1), 39-47.

Romero, E., \& Hernández, M. (2019). Análisis de las causas endógenas y exógenas del abandono escolar temprano: una investigación cualitativa. Educación XXI, 22(1), 263 - 293. doi:10.5944/ educXX1.21351 
Ruiz-Ramírez, R., García-Cué, J., \& Pérez-Olvera, M. (2014). Causas y consecuencias de la deserción escolar en el bachillerato: caso Universidad Autónoma de Sinaloa. Ra Ximhai, 10(5), 51-74. Recuperado de https://www.redalyc.org/html/461/46132134004/

Rumberger, R., \& Lim, S. (2008). Why students drop out of school. California dropout Research Project(5). Recuperado de https://www.issuelab.org/resources/11658/11658.pdf

Salinas, D. (2019). Experiencias y Percepciones de los estudiantes de los programas de educación de adultos en una institución ecuatoriana. Cuenca (Ecuador): Universidad Internacional de la Rioja. Obtenido de https://reunir.unir.net/handle/123456789/9572

Sánchez, D. (2015). La tendencia del abandono escolar en Ecuador: período 1994-2014. Valor agregado, 39-66. Recuperado de https://www.udla.edu.ec/cie/wp-content/uploads/2015/06/ValorAgregado03-Art.-2-S\%C3\%A1nchez-Abandono-escolar.pdf

Sarrate, M. (1997). Educación de adultos: evaluación de centros y experiencias. Madrid: Narcea.

Stake, R.E. (2010). Investigación con estudio de casos. Madrid: Morata. 